Approches interdisciplinaires des enseignements culturels et artistiques dans le cadre de l'enseignement de l'histoire des arts : un modèle complexe, une didactique impossible?

Jean-Charles Chabanne

\title{
OpenEdition
}

Journals

Édition électronique

URL : http://journals.openedition.org/trema/3501

DOI : 10.4000/trema.3501

ISSN : 2107-0997

Éditeur

Faculté d'Éducation de l'université de Montpellier

Édition imprimée

Date de publication : 1 octobre 2016

Pagination : $59-69$

ISBN : 979-10-96627-01-1

ISSN : 1167-315X

Référence électronique

Jean-Charles Chabanne, «Approches interdisciplinaires des enseignements culturels et artistiques dans le cadre de l'enseignement de l'histoire des arts : un modèle complexe, une didactique impossible? », Tréma [En ligne], 45 | 2016, mis en ligne le 01 janvier 2017, consulté le 19 avril 2019. URL : http://journals.openedition.org/trema/3501; DOI : 10.4000/trema.3501

Ce document a été généré automatiquement le 19 avril 2019

Trema 


\section{Approches interdisciplinaires des enseignements culturels et artistiques dans le cadre de l'enseignement de l'histoire des arts : un modèle complexe, une didactique impossible?}

Jean-Charles Chabanne

\section{Introduction}

La présente contribution aborde la notion de «modèle » dans le cadre d'une étude curriculaire de l'introduction d'un nouvel enseignement dans l'enseignement primaire et secondaire, celui de l'histoire des arts (MEN, 2008). Cette innovation peut s'inscrire dans un mouvement de complexité croissante des contenus d'enseignement, corrélative à celle des savoirs savants et des pratiques sociales de référence et à celle des dispositifs d'évaluation impliqués, qui impose aux acteurs une évolution dont le poids est généralement sous-évalué. Quel modèle didactique de l'histoire des arts est proposé ?

\section{Notre approche de la notion de « modèle »}

2 Reprenons la définition qu'I. Delcambre donne de la notion: "formalisation qui permet d'analyser ce qui est enseigné (ou non), ce qui est enseignable (ou non) et les façons dont c'est enseigné. Ainsi, un modèle didactique est une construction théorique à visée descriptive et/ou praxéologique » (2013b, p. 135). 
Concrètement, un modèle se trouve exprimé sous forme compacte (définition) ou schématique (tableau, diagramme) ou développé (parfois sur plusieurs ouvrages, comme par exemple le schéma de la séquence narrative [Adam, 2008]).

La recherche de modèles à des degrés divers de compacité ou de figement pourrait se faire en suivant leurs avatars à diverses étapes de l'élaboration curriculaire: dans les savoirs de référence; dans les savoirs prescrits (textes officiels, programmes, instructions); dans les savoirs préparés pour être enseignés (ressources pour l'enseignant, manuels, préparations...) ; dans les savoirs enseignés (supports remis aux élèves, consignes...) ; dans les savoirs évalués (épreuves d'examen, critères de correction... ); dans les savoirs appris (notes des élèves, réponses aux épreuves d'examen...) ; dans les savoirs analysés (production des chercheurs en didactique...), etc.

5 Ainsi, pour ce qui est d'une synthèse critique des enjeux de l'histoire des arts, on pourrait lire les comptes rendus du Haut Conseil de l'éducation artistique et culturelle, et par exemple celle, fortement critique, de Marc Fumaroli (2007).

6 Les manuels et ouvrages pour l'enseignant sont une forme développée de ce modèle. Plus près des pratiques réelles, on peut utiliser les préparations des enseignants, les affichages, les documents remis aux élèves, comme par exemple les guides de préparation de l'épreuve du Brevet sur les sites de nombreux collèges (où l'on trouve souvent des fiches-guides pour préparer l'analyse d'œuvres). Les savoirs appris sont accessibles via les dossiers, voire certains enregistrements d'exposés oraux, etc.

7 Faute de place ici, nous saisirons le modèle à trois stades :

- dans un modèle-définition qui est la première évocation de cet enseignement nouveau dans le rapport Joutard (1988): "Cette nouvelle approche de l'enseignement de l'histoire des arts se donne pour champ le réseau des objets, des activités artistiques, des pratiques sociales, des institutions »(14e proposition);

- sous sa forme institutionnelle dans le Bulletin officiel (MEN, 2008) ;

- dans le texte qui redéfinit l'épreuve orale du brevet (MEN, 2011).

Le modèle ainsi dessiné témoigne d'un fort degré de complexité. Nous allons passer en revue ces traits, à partir de l'analyse de passages significatifs du texte officiel, dont voici l'intégralité du texte d'ouverture :

L'enseignement de l'histoire des arts est un enseignement de culture artistique partagée. Il concerne tous les élèves. Il est porté par tous les enseignants. Il convoque tous les arts. Son objectif est de donner à chacun une conscience commune : celle d'appartenir à l'histoire des cultures et des civilisations, à l'histoire du monde. Cette histoire du monde s'inscrit dans des traces indiscutables : les œuvres d'art de l'humanité. L'enseignement de l'histoire des arts est là pour en donner les clés, en révéler le sens, la beauté, la diversité et l'universalité (MEN, 2008, p. 1).

\section{Complexité du savoir prescrit}

\section{1. Trait $\mathrm{n}^{\circ} 1$ - « Donner à chacun une conscience commune » : un «trans-éducatif »?}

Un premier trait caractérise le modèle didactique de l'histoire des arts, formulé en termes de finalités générales. L'enseignement de l'histoire des arts s'inscrit dans une préoccupation politique nationale partagée par les deux ministères de l'Éducation et de la Culture depuis de longues années et réactivée encore très récemment par une Feuille de 
route cosignée (MEN \& MCC, 2015) : «Son objectif est de donner à chacun une conscience commune: celle d'appartenir à l'histoire des cultures et des civilisations, à l'histoire du monde " (MEN, 2008, p. 1).

Ce vaste projet que nous proposons d'appeler «transéducatif», pour en souligner l'extrême ambition, s'inscrit dans le cadre de politiques d'éducation internationales (Bamford, 2006) où les arts sont promus comme une composante importante de la New Literacy (Jewitt \& Kress, 2003 ; EU High Level Group of Experts on Literacy, 2012).

11 Il implique de vastes objectifs de construction du collectif, actuellement exprimés dans la visée de refondation de l'École et de l'élaboration d'un Nouveau socle commun de compétences, de connaissances et de culture (MEN, 2015 : Domaine 3, La formation de la personne et du citoyen), qui vise à placer les arts dans une position transversale et fondamentale, afin de repositionner les "humanités et les arts» en regard des «humanités scientifiques » et des techniques.

\section{2. Trait $n^{\circ} 2$ - «II convoque tous les arts » : le trans-esthétique}

12 Une des caractéristiques de l'histoire des arts est qu'elle institue de toutes pièces une "proto-discipline » scolaire qui n'a pas de pendant universitaire. L'expression « histoire des arts » n'appartient pas à la liste des disciplines académiques (Lavin, 1998). Le pluriel « des arts » a été explicitement choisi pour indiquer clairement que les nouveaux savoirs à enseigner ne se contentent pas de transposer l'histoire «de » l'art universitaire : «Le pluriel signifie qu'on sortira largement de la monoculture jusqu'à présent induite par l'autorité envahissante de la peinture et des Beaux-Arts » (Joutard, 1988, ibid.).

Cela est formulé sans ambiguïté dans le texte officiel : «L'histoire des arts intègre l'histoire de l'art par le biais des arts de l'espace, des arts du visuel et des arts du quotidien » (MEN, 2008, p. 1). Elle s'élargit à six grands domaines artistiques (arts de l'espace, du langage, du quotidien, du son, du spectacle vivant, du visuel), mêlant ainsi les arts légitimes à des formes de pratiques culturelles non légitimes selon certains (Fumaroli, 2007).

14 Les approches comparées des arts ont une histoire ancienne (Souriau, 1947 ; Llort Llopart, 2009) et connaissent un fort développement théorique avec l'évolution de l'art contemporain vers la multimodalité (Albers et Sanders, 2010) et la transgression des catégories esthétiques traditionnelles (Lagerroth, Lund et Hedling, 1997). Se développent des recherches en trans-esthétique (Vouilloux, 1997) et les « interart studies » (Collectif, 2006). Mais cela revient à donner comme référence à l'histoire des arts un champ de recherche qui est lui-même en émergence et en construction, entre sciences de la culture et sciences des arts.

\section{3. Trait n 3 - «La multiplicité des approches » : le trans- disciplinaire}

15 Il ne s'agit pas seulement de connaitre ces pratiques, ces œuvres, ou simplement de les rencontrer, mais aussi d'en donner une vue surplombante très vaste : "l'histoire des arts entre en dialogue avec d'autres champs de savoir tels que la culture scientifique et technique, l'histoire des idées, des sociétés, des cultures ou le fait religieux" (Joutard, 1988, ibid.): l'enseignement de l'histoire des arts semble être défini en référence à une vaste anthropologie culturelle critique pluridisciplinaire. 
16 Autre source de complexité, l'histoire des arts s'affirme explicitement comme interdisciplinaire: L'enseignement de l'histoire des arts se fonde sur la reconnaissance de la multiplicité des sources, des influences et des approches; il associe l'analyse du sens à celle des formes, des techniques, des usages (MEN, 2008, p. 1).

17 Ainsi une liste de "thématiques situées au croisement des regards disciplinaires" apparait dans la section consacrée au lycée, relevant de champs anthropologique, historique et social, technique, esthétique (MEN, 2008, p. 13) : de fait, toutes les «sciences de l'art » sont convoquées! À une éducation culturelle conçue comme une expérience sensible des œuvres s'ajoute une initiation à une posture qui emprunte aux sciences sociales son positionnement critique à l'égard d'une éducation aux arts réduite à une pratique de l'admiration: "le nouvel enseignement, attentif aux conditions de la production (technique, matérielle, organisation sociale) n'est pas seulement une histoire des œuvres ou des chefs d'œuvres » (Joutard, 1988, ibid.).

\section{4. Trait $n^{\circ} 4$ - Une conception universaliste et déshistoricisée de l'art : le trans-historique}

18 Il faudrait beaucoup de place pour commenter un autre trait du modèle, qui relève d'un projet éducatif universaliste dont les présupposés seraient à interroger. Sociologues, philosophes et historiens auraient sans doute à redire au postulat d'unicité et de cohérence d'une " histoire » et d'un "monde » au singulier générique. Il en va de même avec les présupposés d'une herméneutique définie à partir de termes comme : «donner les clefs ", "révéler le sens ", etc. Ces présupposés ne relèvent pas seulement d'une théorie esthétique, mais aussi directement d'une didactique car on n'aborde pas de la même façon les œuvres pour en "révéler le sens, la beauté " prédéfinis comme essences ou pour interroger et déconstruire les habitus culturels (Bourdieu, 1979; Esquénazy, 2007 ; Lahire, 2015).

\section{Complexité du didactique}

\section{1. Trait $n^{\circ} 5$ - « Il est porté par tous les enseignants » : l'inter- didactique}

Le modèle didactique de l'histoire des arts fait fi radicalement des cloisonnements disciplinaires: "son enseignement implique la constitution d'équipes de professeurs [...]. L'histoire des arts instaure des situations pédagogiques nouvelles » (MEN, 2008, p. 1).

On pourrait parler ici d'interdidactique pour désigner précisément la difficulté spécifique de se partager un même enseignement en quelque sorte à distance. Car il ne s'agit pas nécessairement d'un enseignement simultané ou intégré (activités d'éveil, Martinand et Host, 1979 ; éducations à : Hasni et Lebeaume, 2010 ; Bousara et Hasni, 2012) mais d'un enseignement partagé à distance, dans lequel les mêmes objets sont enseignés dans des espaces-temps didactiques disjoints: Sans renoncer à leur spécificité, le français, l'histoire-géographie-éducation civique, les langues vivantes et anciennes, la philosophie mais aussi les disciplines scientifiques, économiques, sociales et techniques et l'éducation physique et sportive s'enrichissent de la découverte et de l'analyse des œuvres d'art, des mouvements, des styles et des créateurs (MEN, 2008, p. 1-2). 
21 L'histoire des arts est confiée séparément à toutes les disciplines de l'école, ce qui est une originalité : «l'enseignement de l'histoire des arts [...] fait l'objet d'un volet spécifique dans les programmes des différents champs disciplinaires enseignés aux trois niveaux scolaires » (MEN, 2008, p. 4).

Car mêmes les disciplines qui traditionnellement ont moins naturellement affaire à "l'art » sont invitées à partager ses contenus : Les enseignements artistiques (arts appliqués, arts plastiques, cinéma et audiovisuel, danse, musique, théâtre et arts du cirque) renforcent leurs relations avec les autres disciplines des "humanités", français, histoire-géographie-éducation civique, langues vivantes et anciennes, philosophie et avec les disciplines scientifiques, économiques, sociales et techniques et l'éducation physique et sportive (MEN, 2008, p. 2).

L'évaluation, enfin, peut être assurée par tous : " l'enseignement d'histoire des arts est pris en charge dans le cadre des programmes de toutes les disciplines. À ce titre, tout enseignant peut accompagner des élèves dans la préparation de l'épreuve terminale » (ibid.).

L'histoire des arts participe donc de ces «curriculums chahutés » (Audigier, 2010), où sont bousculées les cloisons disciplinaires, avec les effets qu'on peut imaginer auprès des professionnels dont la formation, l'identité et la pratique se sont forgées à l'intérieur de tout autres cadres. Cela pose des défis épistémologiques et didactiques passionnants. Mais on estime aussi les besoins de recherche et de formation que ce coup de force suppose, tout autant que l'effet de déstabilisation qu'on peut anticiper (De Rohan-Csermak, 2010).

\section{2. Trait $n^{\circ} 6$ - « Le cahier personnel » : pédagogie de projet}

Le modèle de l'histoire des arts s'inscrit en outre dans les tentatives de diffusion de ce qu'on peut appeler pour faire court la pédagogie de projet (Barron, 1998 ; Reverdy, 2013). L'histoire des programmes français montre que plusieurs tentatives ont été faites pour introduire une part de pédagogie de projet dans les pratiques d'enseignement («activités d'éveil » dès 1977, IDD, TPE, PPCP, ECJS... : Éduscol, 2014), la dernière en date étant justement un dispositif qui s'articule à l'histoire des arts, les Parcours d'éducation artistique et culturelle (MEN, 2013) ou les tout récents Enseignements pratiques interdisciplinaires (Éduscol, 2015).

Pour l'équipe enseignante, c'est une ingénierie complexe :

«les professeurs [...] abordent une ou plusieurs cuvres en utilisant les savoirs et les savoirfaire propres à leurs disciplines. [...] Ils les interrogent sous plusieurs angles, opèrent tous les prolongements pertinents et ouvrent au dialogue interdisciplinaire » (MEN, 2008, p. 4).

Pour l'élève, le «projet » s'incarne dans la réalisation d'un document évolutif, de type portfolio, qui requiert l'intégration des savoirs et habiletés dans l'esprit d'une multilittéracie (Kalantzis, Cope et Cloonan, 2010) : L'élève garde mémoire de son parcours dans un "cahier personnel d'histoire des arts". À cette occasion, il met en œuvre ses compétences dans le domaine des TICE, utilise diverses technologies numériques et consulte les nombreux sites consacrés aux arts. Illustré, annoté et commenté par lui, ce cahier personnel est visé par le (ou les) professeur(s) ayant assuré l'enseignement de l'histoire des arts. Il permet le dialogue entre l'élève et les enseignants et les différents enseignants eux-mêmes (MEN, 2008, p. 5).

Dernière difficulté, le cahier d'histoire des arts n'est pas clairement inscrit dans le processus d'évaluation, qui comporte pourtant une part de contrôle continu indépendant : "en classe de troisième, une telle évaluation reste totalement distincte de l'épreuve terminale d'histoire des arts»(MEN, 2011, p. 22). Or on sait qu'une des principales 
objections faites à la pédagogie de projet demeure les difficultés de mesure des acquisitions, voire l'inefficacité (Reverdy, 2013). C'est un des motifs récurrents des résistances qu'on oppose à sa diffusion, sans compter celle qu'on oppose à la notion même d'inter- ou de pluri-disciplinarité (Portella, 1996).

\section{Complexité de l'évaluation}

\section{Trait $n^{\circ} 7$ - «Son objectif est de donner à chacun... " : quels contenus d'apprentissage?}

objet central: le «savoir ». On sait la difficulté à définir la notion même de contenu d'apprentissage (Rey, 2007; Delcambre, 2013a), et Di Rosa (2013) considère que cette question est un «impensé du projet de l'histoire des arts» (p. 46). C'est aussi une question difficile pour la didactique des arts, mais qui n'est que le relais de questions toujours ouvertes en esthétique et philosophie de l'art: celle de la nature de l'expérience esthétique (Genette, 1994,1997; Dewey, 1934/2010; Schaeffer, 2015), de sa nature cognitive (Darsel et Pouivet, 2008), de sa fonction éducative (Kerlan, 2007). Elle est liée à la difficulté à évaluer l'éducation artistique (Kerlan et Erutti, 2008 ; Rouhète, 2008 ; Bamford, 2006). Il en va de même pour une question indirecte, celle des apports du champ de l'éducation et des enseignements artistiques aux « autres » apprentissages (Barton, 2013), dont la littéracie (Flood, Heath \& Lapp, 2008 ; Chabanne, 2015). Ces questions ont une face concrète : sous quelles formes opératoires, selon quels jeux d'apprentissage (Sensevy, 2012) ces «contenus» apparaissent-ils dans les situations? Ont-ils une forme verbale, actionnelle? Quels problèmes-types permettent-ils de résoudre?

Des visées aussi générales que « donner à chacun une conscience commune » ou « donner les clés, en révéler le sens, la beauté, la diversité et l'universalité » ne peuvent en l'état que valoir comme des finalités, les «manières de dire » de Hameline (1983, p. 97), avec leur charge d'implicite à questionner. Mais quelles formes d'évaluation concrètes peuvent-elles rendre possibles? Nous nous attarderons sur trois termes clefs du modèle didactique.

\section{Rencontre}

31 Nous avons par ailleurs (Chabanne \& Dufays, 2011; Chabanne, Parayre et Villagordo, 2012) interrogé ce que peut être une rencontre avec l'œuvre en contexte d'enseignement, pour peu qu'on la considère comme un événement central, du point de vue éducatif (Dewey, 1934/2010), phénoménologique (Schaeffer, 2015), psychologique (Gibson, 2008) ou sociologique (Fleury, 2007; Esquénazy, 2007) et didactique. Tous ces regards convergent pour en faire un événement central, mais aussi l'objet d'une transaction éducative (cela même est à clarifier), d'autant plus qu'ici il s'agit d'une rencontre double, sensible et réfléchie, qui relève de deux dimensions de l'expérience (au double sens d'Erlebnis et de Erfahrung, Schaeffer, 2015).

Cette question complexe est pourtant l'objet de pratiques de référence potentielles : celle savante de la critique d'art (Frangne et Poinsot, 2001) ou ses formes non légitimées que décrit la sociologie de la réception (Soldini et Girel, 2007). Mais comment faire de ces 
pratiques un savoir, et a fortiori un savoir scolaire enseignable, tout en gardant en tête les avertissements de la sociologie quant à la nature fortement différentielle des socialisations esthétiques (Bourdieu et Darbel, 1965 ; Bourdieu, 1979 ; Lahire, 2015).

\section{Culture (personnelle)}

33 La notion de culture personnelle à valeur universelle est elle-même problématique, tout autant que celle de culture humaniste, encore présente dans le texte de 2008, mais disparue de la nouvelle version du Socle commun (MEN, 2015) pour y être remplacée par celle de culture commune. Nous n'avons pas la place ici pour reprendre les débats critiques autour de ces notions relevant encore une fois d'une culture scolaire (Chervel, 1988) marquée d'idéologie (Baldner et Barbaza, 2013).

Un autre angle d'éclairage est donné par l'analyse des critères d'évaluation de l'épreuve terminale, qui révèle en creux une définition de la « culture » comme liste de connaissances encyclopédiques empruntées à plusieurs sciences de l'art : histoire, poïétique, sémiotique, sociologie... (MEN, 2008).

Cette matrice méthodologique qu'on retrouve dans la Grille des critères de l'épreuve orale (MEN, 2011) est, pour l'essentiel, dérivée de celle de l'histoire de l'art académique (Carrier, 1993; Huys et Vernant, 2014). Mais elle ne modélise qu'une partie de l'enjeu didactique, au risque même de s'en détourner.

\section{Amateur éclairé}

La composante à la fois la plus séduisante mais nécessairement la plus problématique du modèle de l'histoire des arts est celle de l'amateur éclairé, « maniant de façon pertinente un premier vocabulaire sensible et technique, maîtrisant des repères essentiels dans le temps et l'espace et appréciant le plaisir que procure la rencontre avec l'art » (MEN, 2008, p. 3).

La notion appartient à l'histoire culturelle de l'art via celle de l'honnête homme de l'époque classique (Lévêque, 1957) mais son lien avec l'art se fait sans doute par le truchement du personnage que Diderot rend fameux comme auteur des Salons (Vouilloux, 1993) et qui sait trouver un équilibre élégant entre l'expression subtile du sensible et la distance du réfléchi. Sans doute aussi faut-il penser à la notion de connoisseur du côté anglosaxon, qui n'est pas le savant (Constable, 1938; Carrier, 2003). On peut dénoncer les ambitions d'un tel programme mais aussi le prendre au sérieux, une fois débarrassé de ses artifices ou illusions (Dumortier, 2012).

Mais la principale difficulté est la modélisation didactique justement : comment l'amateur apprend-il à l'être, si on veut échapper aux illusions de la spontanéité et de l'autarcie ? Où trouver des modèles de l'activité spécifique de l'amateur éclairé: dire l'émotion, les images, les interprétations...? Là encore, la sociologie de l'art montre la multiplicité des acteurs sociaux qui prennent en charge les paroles sur l'œuvre la négociation de son sens et de son évaluation (Heinich, 2009). C'est aussi le discours du public devant une œuvre dans un musée, les échanges des spectateurs à la sortie d'un cinéma, les débats médiatiques autour de l'art contemporain. Mais comment réélaborer en savoir enseignable et évaluable de telles conduites, si cela même est légitime? 
On voit que ce qui est en jeu, effectivement, c'est un projet qui vise à mettre en mouvement les déterminants des conduites et des dispositions, pour diversifier les pratiques culturelles de tous les élèves. Ainsi l'enjeu (légitime) est bien celui d'un projet éducatif réellement transformateur, qui ne peut pas se contenter de déplorer les mécanismes de la distinction ou confondre la neutralité méthodologique et le relativisme axiologique (Rastier, 2013).

\section{Conclusion}

Nous avons cherché à identifier, à partir d'une analyse de textes officiels, quel «modèle didactique » de la proto-discipline histoire des arts était disponible. De toute évidence, le projet adresse aux enseignants de multiples défis. La difficulté à " didactiser le sensible » risque d'amener un enseignement formaliste, aux dépens de ce que le projet présentait de plus passionnant, à savoir la réconciliation dans l'expérience et dans l'éducation à l'École du sensible et du réfléchi (Dewey, 1934/2010). C'est pourtant un beau défi éducatif, tout à la fois théorique et empirique, auquel on peut espérer qu'on donnera le temps de se mettre en place.

\section{BIBLIOGRAPHIE}

Adam, J.-M. (2008). La linguistique textuelle. Introduction à l'analyse textuelle des discours. Paris : Armand Colin.

Albers, P. \& Sanders, J. (Éd.) (2010). Literacies, the arts, and multimodality. Urbana, Ill: National Council of Teachers of English.

Audigier, F. (2010). Les curriculums chahutés. In R. Malet (Éd.), École, médiations et réformes curriculaires : perspectives internationales (pp. 23 37). Bruxelles : De Boeck.

Baldner, J.-M. et Barbaza, A. (Éd.) (2013). Histoire des arts, de la notion à la discipline. Le Français aujourd'hui, 182. Paris : AFEF-Armand Colin.

Bamford, A. (2006). The Wow Factor: Global research compendium on the impact of the arts in education. Münster: Waxmann.

Barron, B. (1998). Doing with understanding: Lessons from research on problem - and projectbased learning. Journal of the Learning Sciences, 7(3/4), 271311.

Barton, G. (2013). The Arts and Literacy: What Does it Mean to be Arts Literate? International Journal of Education \& the Arts, 14(18), 22 p.

Bourdieu, P. (1979). La distinction. Critique sociale du jugement. Paris : Minuit.

Bourdieu, P. et Darbel, A. (1965). L'Amour de l'art. Les musées d'art européens et leur public. Paris : Minuit. 
Bousara, F. et Hasni, A. (2012). L'enseignement par projets et les savoirs disciplinaires en classe de sciences et technologie au Québec compatibilité ou incompatibilité ? Recherches en didactiques. Les Cahiers Théodile, 13, 67-84.

Carrier, D. (1993). Principles of Art History Writing. Pennsylvania: State U.P.

Carrier, D. (2003). In Praise of Connoisseurship. The Journal of Aesthetics and Art Criticism, 61(2), 159-169.

Chabanne, J.-C. (2015). Arts and Literacy: the Specific Contributions of Art to the Development of Multiliteracy. In V. Bozsik (Éd.), Improving Literacy Skills across Learning (CIDREE Yearbook 2015) (pp. 118 135). Budapest: HIERD/CIDREE.

Chabanne, J.-C. et Dufays, J.-L. (2011). Parler et écrire sur les œuvres littéraires et artistiques : contours et enjeux d'une problématique. Repères, 43, 7-29.

Chabanne, J.-C., Parayre, M. et Villagordo, E. (Éd.) (2012). La rencontre avec l'œuvre : éprouver, pratiquer, enseigner les arts et la culture. Paris : L'Harmattan.

Chervel, A. (1988). L'histoire des disciplines scolaires. Réflexions sur un domaine de recherche. Histoire de l'éducation, 38, 59119.

Collectif. (2006). Interart studies - International Research Training Group (En ligne). Berlin: Freie Universität.

Constable, W. (1938). Art History and Connoisseurship. Their Scope and Method. Cambridge: U.P.

Darsel, S. et Pouivet, R. (Éd.) (2008). Ce que l'art nous apprend : les valeurs cognitives dans les arts. Rennes: P. U. de Rennes.

Delcambre, I. (2013a). Contenus d'enseignement et d'apprentissages. In Y. Reuter et al. (Éd.), Dictionnaire des concepts fondamentaux des didactiques (pp. 43 48). Bruxelles : De Boeck.

Delcambre, I. (2013b). Modèle didactique. In Y. Reuter et al. (Éd.), Dictionnaire des concepts fondamentaux des didactiques (pp. 135 138). Bruxelles : De Boeck.

De Rohan-Csermak, H. (2010). La mise en œuvre de l'enseignement de l'histoire des arts en France. In I. Baldriga (Éd.), Il Gusto dei problemi. Rome : RCS Scuola.

Dewey, J. (1934/2010). L'Art comme expérience [Art as experience] (Trad. J. Cometti, rééd. Folio). Paris : Gallimard.

Di Rosa, G. (2013). De la lecture de l'image à l'enseignement de l'histoire des arts. Trajet vers une refonte épistémologique des champs des savoirs. Le Français aujourd'hui, 182, 4352.

Dumortier, J.-L. (2012). Contribuer à la formation d'amateurs éclairés. In J.-C. Chabanne et al., La rencontre avec l'œuvre éprouver, pratiquer, enseigner les arts et la culture (pp. 159-172). Paris :

L'Harmattan.

Éduscol (2014). IDD, TPE, PPCP, ECJS. En ligne : http://eduscol.education.fr/cdi/pratiquespedagogiques/dispositif/tpe

Éduscol (2015). EPI [Enseignements pratiques interdisciplinaires]. En ligne : http:// eduscol.education.fr/pid23187/projet-pluridisciplinaire-a-caractere-professionnel.html

Esquénazy, J.-P. (2007). Sociologie des œuvres. De la production à l'interprétation. Paris : Armand Colin. EU High Level Group of Experts on Literacy (2012). Executive Summary, September 2012. Luxembourg: Publications Office of the European Union. 
Fleury, L. (2007). L'art, l'émotion et les valeurs. Contribution d'une sociologie des émotions à la sociologie de l'art et de la culture. In P. Le Quéau (Éd.), 20 ans de sociologie de l'art, tome 2 (pp. 149 161). Paris : L'Harmattan.

Flood, J., Heath, S. B. \& Lapp, D. (Éd.) (2008). Handbook of research on teaching literacy through the communicative and visual arts. 2. Volume II. New York London: Lawrence Erlbaum Associates.

Frangne, P.-H. et Poinsot, J.-M. (Éd.) (2001). L'Invention de la critique d'art. Rennes : P. U. de Rennes. Fumaroli, M. (2007). Séance du 19/12/2012 [Audition de M. Marc Fumaroli]. In J.-M. (rapporteur général) Pire (Éd.), Rapport annuel du Haut Conseil de l'éducation artistique et culturelle (2006) (pp. 31 36). Paris : Imprimerie du Ministère de la Culture.

Genette, G. (1994). L'œuvre de l'art, I : Immanence et transcendance. Paris : Le Seuil.

Genette, G. (1997). L'œuvre de l'art, II : La Relation esthétique. Paris : Le Seuil.

Gibson, J. (2008). Cognitivism and the Arts. Philosophy Compass, 3(4), 573589.

Hameline, D. (1983). Les objectifs pédagogiques en formation initiale et en formation continue. Paris : ESF.

Hasni, A. et Lebeaume, J. (2010). Enjeux contemporains de l'éducation scientifique et technologique. Ottawa : Presses de l'Université d'Ottawa.

Heinich, N. (2009). Faire voir. L'art à l'épreuve de ses médiations. Paris : Les Impressions Nouvelles.

Huys, V., et Vernant, D. (2014). Histoire de l'art. Théories, méthodes et outils. Paris : Armand Colin.

Jewitt, C. and Kress, G. (Éd.) (2003). Multimodal literacy. New York: Peter Lang.

Joutard, P. (1988). Rapport de la mission de réflexion sur l'enseignement de l'histoire, de la géographie et des sciences sociales. Paris : Ministère de l'Éducation Nationale.

Kalantzis, M., Cope, B. and Cloonan, A. (2010). A Multiliteracies Perspective on the New Literacies. In E. Baker (Éd.), New Literacies. Multiple Perspectives on Research and Practice (pp. 61 87). New York: Guilford Publications.

Kerlan, A. (2007). L'art pour éduquer ? La Tentation esthétique. Contribution philosophique à l'étude d'un paradigme. Laval : P. U. de Laval.

Kerlan, A. et Erutti, R. (2008). Des artistes à l'école : l'expérience esthétique au cœur des apprentissages. In M. Rouhète (Éd.), Évaluer les effets de l'éducation artistique et culturelle (pp. 241 247). Paris : Documentation Française/Centre G. Pompidou.

Lagerroth, U.-B., Lund, H. and Hedling, E. (Éd.) (1997). Interart Poetics: Essays on the Interrelations of the Arts and Media. Amsterdam/Atlanta, GA: Rodopi.

Lahire, B. (2015). Ceci n'est pas qu'un tableau. Essai sur l'art, la domination, la magie et le sacré. Paris : la Découverte.

Lavin, M. (1998). L'histoire des arts. Paris : CNDP/Hachette.

Lévêque, A. (1957). « L'honnête homme » et « l'homme de bien » au XVIIe siècle. PMLA, 72(4), 620 632.

Llort Llopart, V. (2009). Regards croisés des arts. Essai d'esthétique comparée. Paris : L'Harmattan.

Martinand, J.-L. et Host, V. (1979). Effets de l'introduction des activités d'éveil physico-technologiques sur les apprentissages instrumentaux au cycle préparatoire. Fontenay/Paris : ENS de Fontenay/INRP. 
MEN [Ministère de l'Éducation nationale] (2008). Organisation de l'enseignement de l'histoire des arts (école, collège, lycée). Bulletin Officiel $n^{\circ} 32$ du 28 août. Encart.

MEN [Ministère de l'Éducation nationale] (2011). Évaluation de l'histoire des arts à compter de la session 2012. Bulletin Officiel $n^{\circ} 41$ du 10 novembre.

MEN [Ministère de l'Éducation nationale] (2013). Le parcours d'éducation artistique et culturelle (circulaire 2013-073 du 3-5-2013). Bulletin Officiel du 9 mai.

MEN [Ministère de l'Éducation nationale] (2015). Socle commun de connaissances, de compétences et de culture. Journal officiel du 2 avril.

MEN et MCC [Ministère de l'Éducation nationale et Ministère de la culture et de la communication] (2015). Éducation artistique et culturelle, éducation aux médias et à l'information. Paris : MENESR/MCC.

Portella, E. (Éd.) (1996). Entre savoirs. L'interdisciplinarité en acte, enjeux, obstacles, perspectives. Toulouse : Erès.

Rastier, F. (2013). Apprendre pour transmettre. Paris: PUF.

Reuter, Y. (2013). Disciplines scolaires. In Y. Reuter et al. (Éd.), Dictionnaire des concepts fondamentaux des didactiques (pp. 81-85). Bruxelles : De Boeck.

Reuter, Y., Cohen-Azria, C., Daunay, B., Delcambre, I. et Lahanier-Reuter, D. (Éd.) (2013). Dictionnaire des concepts fondamentaux des didactiques. Bruxelles : De Boeck.

Reverdy, C. (2013). Des projets pour mieux apprendre. In Dossier d'actualité nº 82 (pp. 124$)$. Lyon : ENS de Lyon/Ifé.

Rey, B. (2007). Autour du mot « contenu ». Recherche et Formation, 55, 119133.

Rouhète, M. (Éd.) (2008). Évaluer les effets de l'éducation artistique et culturelle. Paris : Documentation française.

Schaeffer, J.-M. (2015). L'expérience esthétique. Paris : Gallimard.

Sensevy, G. (2012). Le jeu comme modèle de l'activité humaine et comme modèle en théorie de l'action conjointe en didactique. SSPS, 7(2), 105132.

Soldini, F. \& Girel, S. (2007). Pour une sociologie du fait artistique. In P. Le Quéau (Éd.), 20 ans de sociologie de l'art, tome 1 (pp. 130 145). Paris : L'Harmattan.

Souriau, E. (1947). La correspondance des arts : éléments d'esthétique comparée. Paris : Flammarion.

Vouilloux, B. (1993). Diderot, Jacques, le Maître, le spectateur et l'amateur - Raconter le tableau, argumenter le goût. Argumentation, 7(1), 89101.

Vouilloux, B. (1997). Langages de l'art et relations transesthétiques. Paris/Tel Aviv : Éditions de l'Éclat.

\section{RÉSUMÉS}

La mise en place d'un enseignement de l'histoire des arts apporte un double défi à relever : d'une part identifier et transposer ou construire des contenus à enseigner qui renvoient à des savoirs de référence multiples et complexes, d'autre part inventer les modalités concrètes d'un enseignement qui impose une approche transdisciplinaire. Existe-t-il un modèle opératoire de cette configuration (inter)disciplinaire nouvelle? 
« History of arts » is a new school subject, introduced in French education since 2009, that is doubly challenging for teachers. First, they have to identify and adapt or build new contents that refer to multiple and complex knowledge. Then, they have to design original instruction devices and techniques, especially since this subject is taught by all teachers whatever their academic discipline, and since there is no existing didactic model.

INDEX

Mots-clés : approches comparatives des enseignements artistiques, éducation artistique et culture, histoire des arts

Keywords : art education., arts history, comparative studies and practices of art education

\section{AUTEUR}

\section{JEAN-CHARLES CHABANNE}

Professeur des universités. Laboratoire Acté (Université Blaise Pascal, Clermont-Ferrand). Institut français de l'Éducation, ENS de Lyon. 